\title{
Patient Characteristics, Safety, and Tolerability with Telaprevir Treatment for HCV in the Clinic: a Retrospective, Multicenter Study
}

\author{
Steven L. Flamm ${ }^{1}$, Paul J. Pockros ${ }^{2}$, Leif Bengtsson ${ }^{3}$ and Mark Friedman ${ }^{3}$ \\ ${ }^{1}$ Northwestern University, Feinberg School of Medicine, Chicago, Illinois, USA; ${ }^{2}$ Scripps Clinic and Scripps Translational Science \\ Institute, La Jolla, California, USA; ${ }^{3}$ Vertex Pharmaceuticals Incorporated, Boston, Massachusetts, USA
}

\begin{abstract}
Background and Aims: There is a paucity of information regarding similarities and differences between patients from the phase 3 studies of telaprevir and those receiving telaprevir in clinical practice. Methods: This retrospective chart review evaluated baseline characteristics and follow-up safety and tolerability data for patients with hepatitis $C$ virus (HCV) infection treated with telaprevir and peginterferon alfa and ribavirin (PR) in clinical practice. Results: In total, 338 charts from patients at four academic and three community US treatment centers who received telaprevir and PR and had at least 12 weeks of follow-up data were included; $62 \%$ were from academic centers and $38 \%$ were from community centers. Of the 338 patients, 269 completed 12 weeks of telaprevir and PR; 32 discontinued due to adverse events. Mean age was 55 years; patients were predominantly white (79.3\%) males (58.9\%) with genotype 1a HCV infection $(61.8 \%) ; 35.5 \%$ were reported to have cirrhosis at baseline; and $55.3 \%$ previously received PR. Hypertension and depression were the most common comorbidities. Patient characteristics outside the per-protocol minimum criteria used in the phase 3 studies of telaprevir were, e.g., hemoglobin, 9.2\%; albumin, 5.3\%; platelets, $11.5 \%$; and neutrophil count, 5.6\%. Adverse events occurred in 329/338 (97.3\%) patients, with anemia, fatigue, nausea, and rash being the most common. Of 38 hospitalizations, 26 were deemed related to telaprevir and PR. Three patients died due to pneumonia, septic shock, and hepatorenal syndrome $(n=1$ each). Conclusions: These findings complement those reported from rigorous, randomized controlled studies with telaprevir-based treatment and provide a general assessment of similarities and/or differences between patients from the phase 3 studies of telaprevir and those treated with telaprevir in clinical practice.

(C) 2014 The Second Affiliated Hospital of Chongqing Medical University. Published by XIA \& HE Publishing Ltd. All rights reserved.
\end{abstract}

Keywords: Hepatitis C virus; Combination drug therapy; Protease inhibitors; Liver diseases.

Abbreviations: $A E$, adverse event; $H C V$, hepatitis C virus; IL28B, interleukin 28B; IRB, institutional review board; $P R$, peginterferon alfa and ribavirin; SAE, serious adverse event; SVR, sustained virologic response.

Received: 11 February 2014; Revised: 16 April 2014; Accepted: 18 April 2014 DOI of original article: 10.14218/JCTH.2014.00007.

Correspondence to: Steven L. Flamm, Northwestern University, Feinberg School of Medicine, 676 N. Saint Clair, Arkes 19-041, Chicago, IL 60611, USA. Tel: $+1-$ 312-695-1681, Fax: +1-312-695-5998, Email: s-flamm@northwestern.edu

\section{Introduction}

Randomized, controlled clinical studies are considered the gold standard for the evaluation of efficacy, safety, and tolerability of pharmaceutical drugs. ${ }^{1,2}$ Such studies allow for rigorous assessment of these parameters in controlled settings that prevent potential confounds when observing a drug's effect(s). ${ }^{3}$ These studies, however, have important characteristics that may limit the generalizability of results, including evaluation of efficacy, safety, and/or tolerability in select patient populations with well-defined and often limited medical comorbidities. These include underrepresentation of the elderly and women, patient selection bias, restrictions regarding concomitant medications, and intensive medical follow-up that is generally not possible in routine clinical care. ${ }^{1,2,4}$ Such limitations have prompted the use of alternative complementary approaches to assess drug treatment outcomes in a clinical practice setting, ${ }^{4}$ including the assessment of medical claims data, establishment of patient registries, patient surveys, and retrospective review of patient charts from a clinical practice setting. Each of these methods has been employed to evaluate the benefits and risks associated with specific treatments for patients with chronic hepatitis $\mathrm{C}$ virus (HCV) infection, ${ }^{5-11}$ thereby providing valuable information on factors influencing treatment adherence, underrepresentation of patients in clinical studies, economic burden of treatment, management of patients with HCV infection, and treatment outcomes.

The efficacy, safety, and tolerability of telaprevir, an NS3/ $4 \mathrm{~A}$ protease inhibitor, in combination with peginterferon alfa and ribavirin (PR) have been evaluated in three randomized, well-controlled, phase 3 clinical studies in patients with genotype 1 chronic HCV infection. The ADVANCE ${ }^{12}$ and ILLUMINATE $^{13}$ studies enrolled treatment-naïve patients, and the REALIZE study ${ }^{14}$ enrolled patients who had not achieved sustained virologic response (SVR) with prior combination therapy with PR. Telaprevir administered in combination with PR for 12 weeks, followed by 12 or 36 weeks of PR alone, was associated with significantly improved SVR rates versus PR alone in treatment-naïve (74\%-79\% versus $46 \%)$ and previously treated $(32 \%-86 \%$ versus $5 \%-$ $22 \%$ ) patients. ${ }^{15}$ Adverse events (AEs) that occurred more often ( $\geqslant 5 \%$ higher frequency) with telaprevir and $P R$ compared with PR alone included rash (56\% vs $34 \%$, respectively), fatigue ( $56 \%$ vs $50 \%)$, pruritus ( $47 \%$ vs $28 \%$ ), nausea ( $39 \%$ vs $28 \%$ ), anemia ( $36 \%$ vs $17 \%)$, diarrhea ( $26 \%$ vs $17 \%$ ), vomiting ( $13 \%$ vs $8 \%)$, hemorrhoids 
Flamm S.L. et al.: Telaprevir treatment for HCV in clinical practice

( $12 \%$ vs $3 \%)$, anorectal discomfort ( $11 \%$ vs $3 \%)$, dysgeusia $(10 \%$ vs $3 \%)$, and anal pruritus ( $6 \%$ vs $1 \%) .12,14,15$ Serious skin reactions, including drug reaction with eosinophilia and systemic symptoms and Stevens-Johnson Syndrome, were reported in $<1 \%$ of patients who received telaprevir and PR. ${ }^{15}$ All three phase 3 studies of telaprevir were controlled, with randomized, double-blind study designs, well-defined inclusion and exclusion criteria, and careful patient follow-up, and each study provided important data on treatment response with telaprevir and PR for patients with $\mathrm{HCV}$ infection. However, as with all randomized, controlled clinical studies, the study designs inherently limit the generalizability of the results to real-world clinical practice settings. In particular, the rigorous entry criteria defined for these studies excluded adolescents and patients older than 70 years, those with human immunodeficiency virus or hepatitis B virus coinfection, and those with blood chemistry and hematology values that were outside normal limits.

To date, there is little information available regarding the similarities and differences between patients enrolled in the phase 3 studies of telaprevir and those receiving telaprevir in a clinical practice setting. This post-marketing, retrospective study reviewed patient charts obtained from academic and community treatment centers and was conducted to evaluate prespecified baseline characteristics and medical follow-up data during the telaprevir treatment phase for patients in the US treated with telaprevir and PR. While not designed to make direct statistical comparisons, this study aimed to assess general similarities and/or differences between the patient populations in the phase 3 studies of telaprevir and those patients treated with telaprevir in real-world clinical practice settings since marketing approval was granted.

\section{Methods}

\section{Study design}

This retrospective study was conducted at four academic and three community treatment centers in the US that were selected based on physicians' clinical experience and involvement with research activities in chronic HCV infection, geographic diversity, and the number of patient charts available for review. Patient charts from academic centers were provided by multiple physicians per site, whereas charts from community centers were provided by a sole physician. Originally, the study protocol planned for 50 consecutive patient charts to be reviewed at each site. However, some sites provided fewer charts than planned and others were permitted to provide more charts to compensate. No site was allowed to provide more than 70 consecutive charts.

The study was conducted in accordance with the current Good Clinical Practice Guidelines of the International Conference on Harmonization, the principles of the Declaration of Helsinki, and local applicable laws and regulations. The institutional review board (IRB) or independent ethics committee of each participating center (Baylor Research Institute IRB, Northwestern University Biomedical IRB, Scripps Office for the Protection of Research Subjects IRB, Temple University Office for Human Subjects Protections IRB, and Western IRB) reviewed and approved the protocol and its revisions. Because no procedures were performed on patients and their personal information was de-identified, the institutional review boards or independent ethics committees of all participating centers waived the need for written informed consent from the patients.

\section{Inclusion criteria}

Charts for the first 50 patients with genotype 1 chronic HCV infection who received telaprevir and PR at one of seven US treatment centers shortly after marketing approval of telaprevir was granted were used (all patients began therapy between May and September 2011). Some sites were permitted to provide more charts (no more than 70 consecutive charts) to compensate for sites that provided fewer than 50 charts. Patient charts were required to include records for at least one dose of telaprevir with PR followed by at least 12 weeks of follow-up. Charts from patients who received treatment with telaprevir during participation in a randomized clinical study were not included.

\section{Data collection}

Case report forms were provided to participating sites, and all appropriate patient data were recorded on these forms. To protect patient confidentiality, data collection was conducted by study site personnel, as designated by the study sponsor, who were trained by the developers of the case report form on the collection of data. If any data required in the case report form were not available for a given patient, this was indicated on the case report form, and those charts were not excluded because of missing data.

Baseline information collected from the patient charts, when available, included sex, age, body mass index, race, ethnicity, medical history, HCV infection history (time since diagnosis, HCV genotype and viral load, interleukin $28 B$ [IL28B] genotype, stage of liver fibrosis, and response to prior HCV treatment), as well as hematology and clinical chemistry values and AEs at baseline and/or on treatment. In some instances, baseline blood work was obtained as many as three months prior to initiation of therapy.

Key on-treatment data extracted from the patient charts, when available, included telaprevir dosing, including any modifications for anemia, rash, pruritus, or anorectal adverse events; PR dosing, including modifications based on the above-listed events; transfusions or administration of erythropoietin-stimulating agents; concomitant medications; and AEs and serious AEs (SAEs). Modifications to telaprevir and/or PR dosing were made at the discretion of the treatment center. Data regarding hospitalizations, clinical chemistry, hematology, and HCV RNA levels were also collected, when available.

Available information for AEs, as interpreted by study site personnel, included classification as serious or not serious, dates of onset and resolution, severity, causal relation to study drug(s), action taken, concomitant medications or other treatments given, and outcomes as typically reported during the conduct of clinical studies.

\section{Pooled results from the phase 3 clinical studies of telaprevir}

Results from 1,797 treatment-naïve and -experienced patients who were assigned to 8 weeks ( $n=364$; not an approved treatment regimen) or 12 weeks ( $n=1433$; approved treatment regimen) of treatment with telaprevir and PR followed by 12 or 36 weeks of PR alone (total 
treatment duration of 24 or 48 weeks) were pooled, when possible, from the three phase 3 studies of telaprevir. In these phase 3 studies, telaprevir was administered at 750 mg every 8 hours; the peginterferon alfa-2a dose was 180 micrograms per week, and the ribavirin dose was $1,000 \mathrm{mg}$ per day (patients weighing $<75 \mathrm{~kg}$ ) or $1,200 \mathrm{mg}$ per day (patients weighing $\geqslant 75 \mathrm{~kg}$ ). The post hoc review of data for these patients aimed to match information collected in the chart review and included baseline demographic characteristics (sex, age, body mass index, race, and ethnicity), medical history, HCV infection history (time since diagnosis, HCV genotype and baseline viral load, IL28B genotype, stage of liver fibrosis at baseline, and response to prior HCV therapy), as well as hematology and clinical chemistry values and AEs at baseline and/or on treatment.

\section{Data analysis}

Whenever possible, general comparisons were made with pooled data from the phase 3 studies of telaprevir, but no statistical analyses were performed.

\section{Results}

In total, 338 patient charts containing records for patients receiving telaprevir and PR with at least 12 weeks of follow-up were included in the study. Of these, $210(62 \%)$ charts were from patients treated at academic treatment centers, and $128(38 \%)$ were from patients treated at community treatment centers. Of these 338 patients, 269 (79.6\%) had completed the recommended 12 weeks of treatment with telaprevir and PR. The most common reason for treatment discontinuation, which occurred in $69(20.4 \%)$ patients, was AEs (Table 1). Almost half of the discontinuations in the clinical practice group $(n=32)$ were attributable to AEs.

Baseline and demographic characteristics of the patients from clinical practice and patients from the phase 3 studies of telaprevir are shown in Table 2. Patients included in the clinical practice group had a mean age of 55 years, were predominantly male $(58.9 \%)$ and white $(79.3 \%)$, and had

Table 1. Reasons for discontinuation of telaprevir-based treatment for patients in the clinical practice group

\begin{tabular}{|c|c|}
\hline & $\begin{array}{l}\text { Total Patients } \\
\quad(n=338)\end{array}$ \\
\hline $\begin{array}{l}\text { Patients who completed } 12 \\
\text { weeks of } T / P R, n(\%)\end{array}$ & $269(79.6)$ \\
\hline $\begin{array}{l}\text { Patients who discontinued } \\
\text { treatment, n (\%) }\end{array}$ & $69(20.4)$ \\
\hline \multicolumn{2}{|c|}{ Reason for treatment discontinuation, $\mathrm{n}(\%)$} \\
\hline Adverse event & $32(9.5)$ \\
\hline Physician decision & $18(5.3)$ \\
\hline Other & $9(2.7)$ \\
\hline Patient refused further dosing & $4(1.2)$ \\
\hline Lost to follow-up & $3(0.9)$ \\
\hline Death & $1(0.3)$ \\
\hline Noncompliance with study drug & $1(0.3)$ \\
\hline Other noncompliance & $1(0.3)$ \\
\hline
\end{tabular}

$P R$, peginterferon and ribavirin. been diagnosed with $\mathrm{HCV}$ infection for a mean of nine years; $35.5 \%$ of patients had cirrhosis at baseline. Assessment of HCV RNA genotype showed that genotype 1a was predominant $(61.8 \%)$. Only $30.2 \%$ of patients in the clinical practice group had data available for IL28B genotype (8.0\% CC, $14.5 \% \mathrm{CT}$, and $7.7 \% \mathrm{TT}$ ). Medical comorbidities were common, with hypertension (36.4\%) and depression $(30.2 \%)$ being the two most frequently observed comorbid conditions in this patient population (Table 3).

Of the 338 patients in the clinical practice group, 187 (55.3\%) had been previously treated with PR therapy; of those, $80(23.7 \%)$ had a null response to prior treatment. Most patient charts included information regarding the frequency of telaprevir dosing; 257 (76.0\%) and 73 $(21.6 \%)$ patients received telaprevir three times daily and twice daily, respectively.

Selected baseline hematology and clinical chemistry variables, including mean hemoglobin, albumin, total bilirubin levels, platelet, and absolute neutrophil counts, are shown in Table 4 for patients in the clinical practice group and in the phase 3 studies of telaprevir. In the clinical practice group, 31 $(9.2 \%)$ patients had baseline hemoglobin levels below the lower limits required per protocol (130 g/L for males; $120 \mathrm{~g} / \mathrm{L}$ for females) for enrollment in the phase 3 studies of telaprevir. The mean reductions in hemoglobin levels for patients in the clinical practice group and in the phase 3 studies of telaprevir over the first 12 weeks of treatment are shown in Fig. 1. Overall, mean reductions during the first four weeks and the entire 12 weeks of treatment were similar. In the clinical practice group, patients who began treatment with telaprevir and PR with other baseline hematology and clinical chemistry values below the per-protocol minimum criteria set for the phase 3 studies of telaprevir were as follows: 18 $(5.3 \%)$ patients with albumin levels $<33 \mathrm{~g} / \mathrm{L}, 9(2.7 \%)$ patients with total bilirubin levels $>38 \mu \mathrm{mol} / \mathrm{L}, 39(11.5 \%)$ patients with platelet counts $<90 \times 10^{9} / \mathrm{L}, 19(5.6 \%)$ patients with absolute neutrophil counts $<1.5 \times 10^{9} / \mathrm{L}$, and three $(0.9 \%)$ patients with thyroid-stimulating hormone levels $>5.6 \mathrm{mIU} / \mathrm{L}$ (Table 4 ). Among patients who had platelet counts $<90 \times 10^{9} / \mathrm{L}, 11(28.2 \%)$ had platelet counts $<60$ $\times 10^{9} /$ L.

AEs experienced by at least $20 \%$ of patients are summarized in Table 5. Overall, AEs were reported by 329 of the 338 $(97.3 \%)$ patients in the clinical practice group. Four patients in the clinical practice group experienced life-threatening AEs (defined as a patient being at immediate risk of death from the event as it occurred) that included hepatorenal syndrome, rectal cancer, and pneumonia in three patients $(n=1$ each) and mental status change, respiratory distress, and septic shock in one patient. Three $(0.9 \%)$ of these four patients died, with their deaths attributed separately to pneumonia, septic shock, and hepatorenal syndrome. Thirty-nine patients $(11.5 \%)$ reported AEs rated as severe in intensity, and the most common (occurring in $\geqslant 2$ patients) were anemia $(n=15,4.4 \%)$, rash $(n=6,1.8 \%)$, thrombocytopenia $(n=4,1.2 \%)$, neutropenia $(n=2,0.6 \%)$, pruritus $(n=2,0.6 \%)$, anxiety $(n=2,0.6 \%)$, and syncope $(n=2$, $0.6 \%$ ). AEs experienced by $\geqslant 20 \%$ of patients that occurred at a rate of at least $5 \%$ higher in the clinical practice group than the Phase 3 pooled data were anemia and dyspepsia. Those that occurred at least $5 \%$ higher in the Phase 3 pooled data than the clinical practice group were pruritus, headache, pyrexia, and influenza-like illness. A total of 70 SAEs occurred in $40(11.8 \%)$ patients in the clinical practice group; 48 of 
Flamm S.L. et al.: Telaprevir treatment for HCV in clinical practice

Table 2. Patient baseline and demographic characteristics

\begin{tabular}{|c|c|c|}
\hline Characteristic & $\begin{array}{l}\text { Clinical practice group } \\
\qquad(\mathrm{n}=338)\end{array}$ & $\begin{array}{c}\text { Phase } 3 \text { pooled data }{ }^{a} \\
(n=1797)\end{array}$ \\
\hline \multicolumn{3}{|l|}{ Prior therapy with $\mathrm{PR}, \mathrm{n}(\%)$} \\
\hline Treatment naïve & $148(43.8)$ & $1267(70.5)$ \\
\hline Treatment experienced & $187(55.3)$ & $530(29.5)$ \\
\hline Prior relapser & $61(18.0)$ & $286(15.9)$ \\
\hline Partial responder & $19(5.6)$ & $97(5.4)$ \\
\hline Null responder & $80(23.7)$ & $147(8.2)$ \\
\hline Unknown response to PR & $27(8.0)$ & 0 \\
\hline Unknown treatment experience & $3(0.9)$ & 0 \\
\hline Mean age, years (SD) & $55(9.1)$ & $49(9.8)$ \\
\hline Male, n (\%) & $199(58.9)$ & $1122(62.4)$ \\
\hline Mean BMI, kg/m² (SD) & $29(5.9)$ & $27(5.3)$ \\
\hline \multicolumn{3}{|l|}{ Race, n (\%) } \\
\hline White & $268(79.3)$ & $1565(87.1)$ \\
\hline Black & $35(10.4)$ & $158(8.8)$ \\
\hline Other & $27(8.0)$ & $61(3.4)$ \\
\hline Not collected per local regulations & $7(2.1)$ & $13(0.7)^{b}$ \\
\hline Unknown & $1(0.3)$ & 0 \\
\hline \multicolumn{3}{|l|}{ Ethnicity, n (\%) } \\
\hline Hispanic or Latino & $17(5.0)$ & $185(10.3)$ \\
\hline Not Hispanic or Latino & $273(80.8)$ & $1599(89.0)$ \\
\hline Not collected per local regulations & $43(12.7)$ & $13(0.7)^{b}$ \\
\hline Unknown & $5(1.5)$ & 0 \\
\hline \multicolumn{3}{|l|}{ HCV genotype subtype, n (\%) } \\
\hline $1 a$ & $209(61.8)$ & $1103(61.4)$ \\
\hline $1 b$ & $64(18.9)$ & $676(37.6)$ \\
\hline Unknown/other & $65(19.2)$ & $18(1.0)$ \\
\hline \multicolumn{3}{|l|}{ IL28B genotype, $\mathrm{n}(\%)$} \\
\hline $\mathrm{CC}$ & $27(8.0)$ & Only \\
\hline CT & $49(14.5)$ & partially \\
\hline$\pi$ & $26(7.7)$ & assessed \\
\hline Unknown & $236(69.8)$ & \\
\hline Mean HCV RNA $\log _{10}, \mathrm{IU} / \mathrm{mL}$ (SD) & $6.2(0.9)$ & $6.4(0.7)$ \\
\hline Mean time since HCV diagnosis, years (SD) & $9(8.2)$ & $7(6.6)$ \\
\hline \multicolumn{3}{|l|}{ Stage of fibrosis/cirrhosis, $\mathrm{n}(\%)$} \\
\hline No or minimal fibrosis & $61(18.0)$ & $528(29.4)$ \\
\hline Portal fibrosis & $59(17.5)$ & $705(39.2)$ \\
\hline Bridging fibrosis & $56(16.6)$ & $317(17.6)$ \\
\hline Cirrhosis & $120(35.5)$ & 247 (13.7) \\
\hline Unknown & $42(12.4)$ & 0 \\
\hline
\end{tabular}

${ }^{a}$ Includes patients who were assigned to 8 or 12 weeks of treatment with telaprevir in combination with peginterferon alfa and ribavirin.

${ }^{b}$ From the ILLUMINATE study. ${ }^{13}$

BMI, body mass index; HCV, hepatitis C virus; IL28B, interleukin 28B; N/A, not applicable; PR, peginterferon alfa and ribavirin; RNA, ribonucleic acid; SD, standard deviation.

these SAEs were considered to be related to treatment in 31 $(9.2 \%)$ patients. The most commonly reported SAEs were anemia and rash, occurring in seven $(2.1 \%)$ and six $(1.8 \%)$ patients, respectively (Table 6$)$. There was also one $(0.3 \%)$ patient with an SAE of drug reaction with eosinophilia and systemic symptoms; no SAEs of Stevens-Johnson syndrome or toxic epidermal necrolysis were reported. Fifty-five percent $(n=22)$ of the patients who experienced SAEs had documented baseline cirrhosis. SAEs were reported in $18 \%$ of patients with documented cirrhosis at baseline. Hospitalizations during the treatment period occurred in 38 (11.2\%) patients; of these hospitalizations, 26 were determined to be related to telaprevir and PR treatment, with 11 due to the need for blood transfusions. 
Flamm S.L. et al.: Telaprevir treatment for HCV in clinical practice

Table 3. Comorbid medical conditions reported in $\geqslant 5 \%$ of patients in either the retrospective study or in the phase 3 studies

\begin{tabular}{lcc}
\hline Condition, $\mathrm{n}(\%)$ & $\begin{array}{c}\text { Clinical } \\
\text { practice group } \\
(\mathrm{n}=338)\end{array}$ & $\begin{array}{c}\text { Phase } 3 \\
\text { pooled data } \\
(\mathrm{n}=1267)\end{array}$ \\
\hline Hypertension & $123(36.4)$ & $288(22.7)$ \\
Depression & $102(30.2)$ & $217(17.1)$ \\
Gastroesophageal reflux & $50(14.8)$ & $142(11.2)$ \\
disease & $49(14.5)$ & $113(8.9)$ \\
Anxiety & $34(10.1)$ & $180(14.2)$ \\
Insomnia & $34(10.1)$ & $5(0.4)$ \\
Hepatic cirrhosis & $31(9.2)$ & $53(4.2)$ \\
Hypothyroidism & $24(7.1)$ & $53(4.2)$ \\
Asthma & $24(7.1)$ & $10(0.8)$ \\
Thrombocytopenia & $23(6.8)$ & $46(3.6)$ \\
Diabetes mellitus & $23(6.8)$ & $33(2.6)$ \\
Obesity & $20(5.9)$ & $48(3.8)$ \\
Arthritis & $18(5.3)$ & $8(0.6)$ \\
Esophageal varices & $17(5.0)$ & $120(9.5)$ \\
Back pain & $10(3.0)$ & $104(8.2)$ \\
Fatigue & $10(3.0)$ & $67(5.3)$ \\
Hemorrhoids & $8(2.4)$ & $94(7.4)$ \\
Seasonal allergy & $8(2.4)$ & $66(5.2)$ \\
Hysterectomy & $6(1.8)$ & $102(8.1)$ \\
Arthralgia & $4(1.2)$ & $94(7.4)$ \\
Headache & $3(0.9)$ & $83(6.6)$ \\
Drug hypersensitivity & $10(3.0)$ & $94(7.4)$ \\
Drug abuse & & \\
\hline & &
\end{tabular}

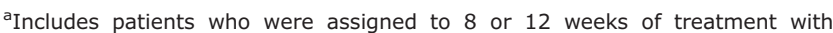
telaprevir and peginterferon alfa and ribavirin.

' Includes data for treatment-naïve patients enrolled in the ADVANCE and ILLUMINATE studies only. Data for treatment-experienced patients enrolled in the REALIZE study were excluded because the source data collection and dictionary encoding methods for comorbid medical conditions were different for the REALIZE study, resulting in different classifications for the medical conditions and preventing pooling of the data.

Telaprevir treatment was interrupted in $17(5 \%)$ patients in the clinical practice group due to AEs. Telaprevir doses were modified because of anemia in five (1.5\%) patients, rash in 10 $(3.0 \%)$ patients, pruritus in five $(1.5 \%)$ patients, anorectal disorder in zero patients, and other reasons in 20 (5.9\%) patients. Peginterferon doses were modified because of anemia in $21(6.2 \%)$ patients, rash in seven $(2.1 \%)$ patients, pruritus in $2(0.6 \%)$ patients, anorectal disorder in one $(0.3 \%)$ patient, and other reasons in $57(16.9 \%)$ patients. Ribavirin doses were modified due to anemia in $153(45.3 \%)$ patients, rash in $21(6.2 \%)$ patients, pruritus in seven $(2.1 \%)$ patients, anorectal disorder in three $(0.9 \%)$ patients, and other reasons in $39(11.5 \%)$ patients. During treatment with telaprevir and PR, $114(33.7 \%)$ and $23(6.8 \%)$ patients received erythropoietin and blood transfusions, respectively.

\section{Discussion}

This retrospective study of real-life clinical patients who received telaprevir and PR for chronic HCV infection provides valuable insight into the baseline characteristics and safety and tolerability outcomes of the population of treated patients that may not be represented within the controlled setting of a randomized study. The composite baseline patient profiles in this current study may be considered more difficult to treat than those in the phase 3 studies, as many patients were older (mean age, 55 vs 49 years), a greater proportion had cirrhosis ( $36 \%$ vs $14 \%$ ), and more patients were treatment experienced ( $55 \%$ vs $29 \%$ ). In addition, a substantial percentage of patients treated with telaprevir and PR in the clinical practice group would not have met the per-protocol minimum baseline clinical chemistry and hematology criteria for inclusion in the phase 3 studies. Although this would suggest that such patients may have been excluded from the phase 3 studies, it is important to note that there were occasional protocol deviations in the phase 3 studies regarding clinical chemistry and hematology inclusion criteria. In the clinical practice group, the treatment of patients with relatively low levels of hemoglobin, platelets, and albumin suggests that physicians in clinical practice may feel an urgency to treat these patients. Additionally, patients included in the current study had comorbid conditions, including depression, hypothyroidism, asthma, thrombocytopenia, and esophageal varices, which potentially could have excluded them from participation in the phase 3 studies of telaprevir. Many of these observed differences in patient characteristics likely contributed to some of the differences observed in rates of AEs, particularly anemia.

Since the approval of telaprevir in 2011, telaprevir-based therapy has been purported to be less well tolerated than was expected given the data from the phase 3 studies. In the current study, almost $80 \%$ of patients completed the recommended 12-week period of telaprevir and PR treatment, with $10 \%$ of patients discontinuing treatment because of an AE. This suggests that AEs experienced by patients in a clinical practice setting were generally manageable, and most of the reported AEs were characterized as mild or moderate. Anemia, rash, and pruritus were among the most common AEs associated with the use of telaprevir, which is consistent with data from the phase 3 studies. ${ }^{12-14}$ The incidence of pruritus, headache, pyrexia, and influenza-like illness was higher in patients in the phase 3 studies, while the incidence of anemia and dyspnea was higher in patients in the clinical practice group. While $60 \%$ of clinical practice patients experienced anemia as an $A E, 33 \%$ of patients did so in the phase 3 studies. However, the proportion of patients who reported anemia as an SAE was similar between both groups ( $2.1 \%$ in the clinical practice group vs $1.8 \%$ in the phase 3 studies). In addition, while the pattern of reduction in hemoglobin levels during telaprevir treatment was similar between both groups, the mean levels were lower in patients in the clinical practice group.

It appears that incident anemia was managed successfully in most cases with ribavirin dose reductions and erythropoietin supplementation; blood transfusions were thought to be necessary occasionally. Relatively few SAEs $(n=15)$ were associated with anemia, pruritus, or rash.

In the clinical practice group, $35.5 \%$ of patients had cirrhosis at baseline. Of the 40 patients who reported SAEs, more than half had existing cirrhosis. The increased rate of SAEs observed in patients with cirrhosis is consistent with preliminary findings from the French Compassionate Use of Protease Inhibitors in Cirrhosis (CUPIC) study, an independent patient registry established in Europe to observe patients undergoing HCV treatment in the clinical setting. ${ }^{10,16}$ 
Flamm S.L. et al.: Telaprevir treatment for HCV in clinical practice

Table 4. Selected mean baseline hematology and clinical chemistry variables

\begin{tabular}{|c|c|c|}
\hline Variable & $\begin{array}{l}\text { Clinical practice group } \\
\qquad(\mathrm{n}=338)\end{array}$ & $\begin{array}{l}\text { Phase } 3 \text { pooled data }{ }^{a} \\
\quad(n=1797)\end{array}$ \\
\hline \multicolumn{3}{|l|}{ Hemoglobin, g/L } \\
\hline $\mathrm{n}$ & 299 & 1795 \\
\hline Mean (SD) & $144(16.2)$ & $150(13.0)$ \\
\hline Minimum, maximum & 49,184 & 105,228 \\
\hline $\begin{array}{l}\text { Patients with levels }<\text { per-protocol requirement for phase } 3 \text { study entry, } \\
\mathrm{n}(\%)\end{array}$ & $31(9.2)$ & $\mathrm{N} / \mathrm{A}$ \\
\hline \multicolumn{3}{|l|}{ Albumin, g/L } \\
\hline $\mathrm{n}$ & 287 & 1797 \\
\hline Mean (SD) & $40(4.7)$ & $44(3.1)$ \\
\hline Minimum, maximum & 12,50 & 32,52 \\
\hline $\begin{array}{l}\text { Patients with levels }<\text { per-protocol requirement for phase } 3 \text { study entry, }{ }^{c} \\
\mathrm{n}(\%)\end{array}$ & $18(5.3)$ & N/A \\
\hline \multicolumn{3}{|l|}{ Total bilirubin, $\mu \mathrm{mol} / \mathrm{L}$} \\
\hline $\mathrm{n}$ & 285 & 1797 \\
\hline Mean (SD) & $14(10.3)$ & $10(5.0)$ \\
\hline Minimum, maximum & 3,128 & 2,50 \\
\hline $\begin{array}{l}\text { Patients with levels }>\text { per-protocol requirement for phase } 3 \text { study entry, }{ }^{d} \\
\mathrm{n}(\%)\end{array}$ & $9(2.7)$ & $\mathrm{N} / \mathrm{A}$ \\
\hline \multicolumn{3}{|l|}{ Platelet count, $10^{9} / \mathrm{L}$} \\
\hline $\mathrm{n}$ & 296 & 1790 \\
\hline Mean (SD) & $174(72.5)$ & $238(73.0)$ \\
\hline Minimum, maximum & 39,500 & 82,655 \\
\hline $\begin{array}{l}\text { Patients with levels }<\text { per-protocol requirement for phase } 3 \text { study entry, } \\
\mathrm{n}(\%)\end{array}$ & $39(11.5)$ & N/A \\
\hline \multicolumn{3}{|l|}{ Absolute neutrophil count, $10^{9} / \mathrm{L}$} \\
\hline $\mathrm{n}$ & 257 & 1794 \\
\hline Mean (SD) & $3(1.3)$ & $4(1.4)$ \\
\hline Minimum, maximum & 1,8 & 1,17 \\
\hline $\begin{array}{l}\text { Patients with levels }<\text { per-protocol requirement for phase } 3 \text { study entry, } \\
\mathrm{n}(\%)\end{array}$ & $19(5.6)$ & N/A \\
\hline \multicolumn{3}{|l|}{ Thyroid stimulating hormone, mIU/L } \\
\hline $\mathrm{n}$ & 166 & 1797 \\
\hline Mean (SD) & $2(7.3)$ & $2(1.0)$ \\
\hline Minimum, maximum & 0,95 & 0,13 \\
\hline $\begin{array}{l}\text { Patients with levels }>\text { per-protocol requirement for phase } 3 \text { study entry, }{ }^{9} \\
\mathrm{n}(\%)\end{array}$ & $3(0.9)$ & N/A \\
\hline
\end{tabular}

${ }^{a}$ Includes patients who were assigned to 8 or 12 weeks of treatment with telaprevir in combination with peginterferon alfa and ribavirin.

ber-protocol requirement for phase 3 study entry: $\geqslant 130 \mathrm{~g} / \mathrm{L}$ (males); $\geqslant 120 \mathrm{~g} / \mathrm{L}$ (females)

cPer-protocol requirement for phase 3 study entry: $\geqslant 33 \mathrm{~g} / \mathrm{L}$

${ }^{\mathrm{d}}$ Per-protocol requirement for phase 3 study entry: $\leqslant 38 \mu \mathrm{mol} / \mathrm{L}$

eper-protocol requirement for phase 3 study entry: $\geqslant 90 \times 10^{9} / \mathrm{L}$

fPer-protocol requirement for phase 3 study entry: $\geqslant 1.5 \times 10^{9} / \mathrm{L}$

${ }^{9}$ Per-protocol requirement for phase 3 study entry: $\leqslant 5.6 \mathrm{mIU} / \mathrm{L}$

N/A, not applicable; SD, standard deviation.

In the HCV-TARGET study, a similar registry established in the US, patients with cirrhosis were at increased risk for discontinuing treatment early because of AEs. ${ }^{6,17}$ These data highlight the need for careful and routine monitoring of patients with cirrhosis in the clinical setting.

Although an attempt was made to collect available data with respect to on-treatment efficacy outcomes, an insufficient number of patients had been assessed at the relevant time points or had an incomplete history for treatment outcomes, which prevented analysis and interpretation of the data. However, the CUPIC study in Europe ${ }^{10}$ and the HCVTARGET study in the US ${ }^{6,17,18}$ have been designed to collect data regarding on-treatment efficacy outcomes in the clinical setting.

Retrospective reviews of patient charts provide information from readily accessible, existing data, are relatively 
Flamm S.L. et al.: Telaprevir treatment for HCV in clinical practice

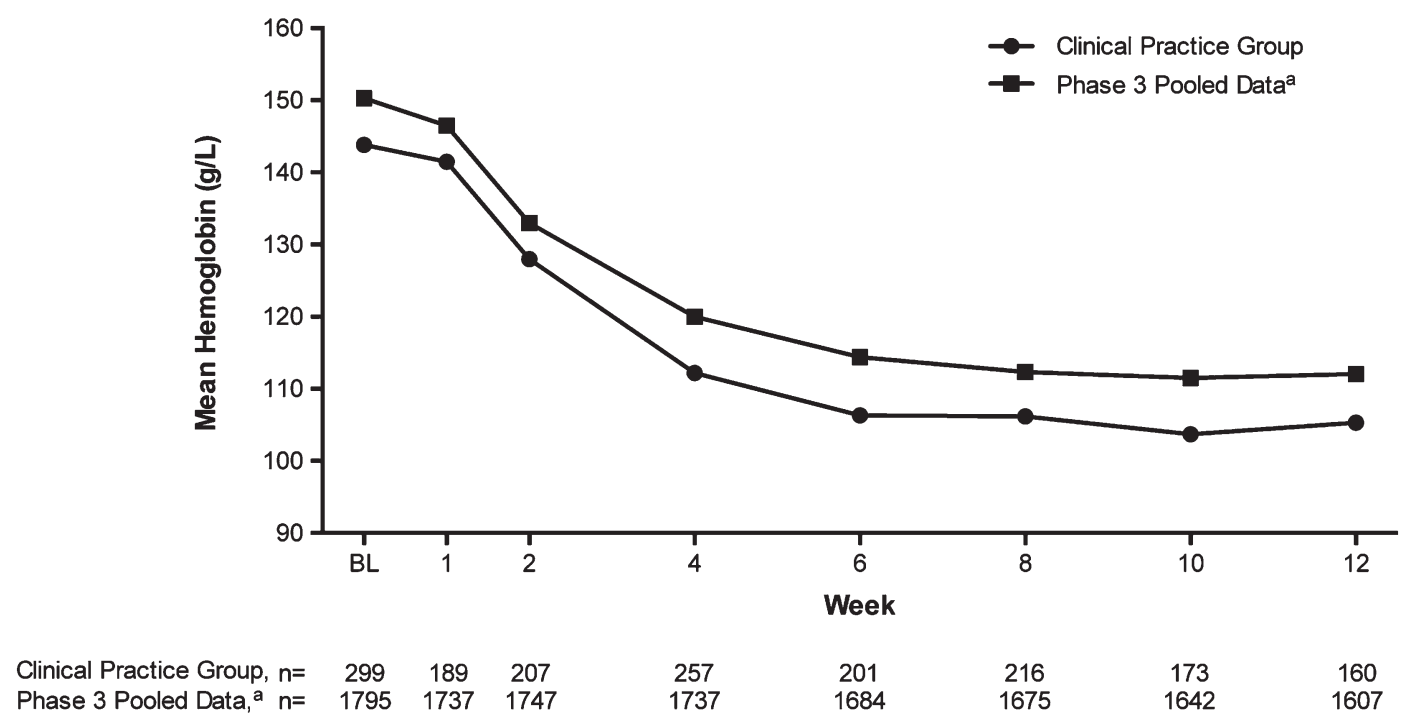

Fig. 1. Mean reductions in hemoglobin levels during telaprevir-based treatment. Data for patients in the clinical practice group and in the phase 3 studies of telaprevir are plotted together for illustrative purposes; however, no statistical analyses were performed for comparison. ${ }^{a}$ Includes patients who were assigned to 8 or 12 weeks of treatment with telaprevir in combination with peginterferon alfa and ribavirin. BL, baseline.

inexpensive to conduct, may be important in hypothesis generation for prospective studies, and may allow the study of rare occurrences or conditions where there is a long latency period between exposure and disease. However, limitations inherent to retrospective studies include incomplete documentation, problems in interpreting and verifying information (e.g., differences in terminology used by clinicians), variance in the quality of information as recorded by clinicians, and difficulty in determining cause and effect between treatment exposure and outcomes. ${ }^{19,20}$ The current study was performed at only seven sites that, despite the diversity in physicians' clinical experience and involvement with research activities in chronic HCV infection, the geography, and the

Table 5. Adverse events experienced by $\geqslant 20 \%$ of patients in either the retrospective study or phase 3 studies during telaprevir-based treatment

\begin{tabular}{lcc}
\hline Event, $\mathrm{n}(\%)$ & $\begin{array}{c}\text { Clinical } \\
\text { practice group } \\
(\mathrm{n}=338)\end{array}$ & $\begin{array}{c}\text { Phase 3 } \\
\text { pooled data } \\
(\mathrm{n}=1797)\end{array}$ \\
\hline Any AE & $\mathbf{3 2 9 ( \mathbf { 9 7 . 3 } )}$ & $\mathbf{1 7 7 3 ( \mathbf { 9 8 . 7 } )}$ \\
Anemia & $204(60.4)$ & $590(32.8)$ \\
Fatigue & $181(53.6)$ & $998(55.5)$ \\
Nausea & $147(43.5)$ & $704(39.2)$ \\
Rash & $122(36.1)$ & $597(33.2)$ \\
Pruritus & $98(29.0)$ & $840(46.7)$ \\
Dyspnea & $76(22.5)$ & $242(13.5)$ \\
Diarrhea & $75(22.2)$ & $458(25.5)$ \\
Insomnia & $74(21.9)$ & $458(25.5)$ \\
Headache & $54(16.0)$ & $657(36.6)$ \\
Pyrexia & $40(11.8)$ & $392(21.8)$ \\
Influenza-like illness & $14(4.1)$ & $516(28.7)$ \\
\hline
\end{tabular}

${ }^{a}$ Includes patients who were assigned to 8 or 12 weeks of treatment with telaprevir in combination with peginterferon alfa and ribavirin. $A E$, adverse event. number of patient charts available for review, may not fully represent all treatment centers in which patients with chronic $\mathrm{HCV}$ infection are treated. Additionally, patients included in the clinical practice group were not randomly selected. Data were collected by site personnel who were trained by the developers of the case record form to record all appropriate patient data. However, uniform approaches of data entry may not have occurred at all sites, and charts did not always contain complete patient information, resulting in some missing data. For example, while obesity was reported as a comorbidity in $6.8 \%$ of patients in the clinical practice group, the mean body mass index was $29 \mathrm{~kg} / \mathrm{m}^{2}$, highlighting the lack of uniformity in approaches to data entry. In some instances, baseline blood work was obtained as many as three months prior to the initiation of therapy. Furthermore, while most sites were associated with academic hospitals,

Table 6. Serious adverse events during telaprevir-based treatment experienced by $\geqslant 0.5 \%$ of patients in either the retrospective study or in the phase 3 studies

\begin{tabular}{lcc}
\hline Event, $\mathrm{n}(\%)$ & $\begin{array}{c}\text { Clinical } \\
\text { practice group } \\
(\mathrm{n}=338)\end{array}$ & $\begin{array}{c}\text { Phase } 3 \\
\text { pooled data } \\
(\mathrm{n}=1797)\end{array}$ \\
\hline Patients with any SAEs & $\mathbf{4 0 ( 1 1 . 8 )}$ & $\mathbf{9 9 ( 5 . 5 )}$ \\
Anemia & $7(2.1)$ & $33(1.8)$ \\
Rash & $6(1.8)$ & $6(0.3)$ \\
Syncope & $3(0.9)$ & $2(0.1)$ \\
Anxiety & $2(0.6)$ & 0 \\
Dehydration & $2(0.6)$ & $2(0.1)$ \\
Fatigue & $2(0.6)$ & 0 \\
Hypokalemia & $2(0.6)$ & 0 \\
Pruritus & $2(0.6)$ & $2(0.1)$ \\
\hline
\end{tabular}

ancludes patients who were assigned to 8 or 12 weeks of treatment with telaprevir in combination with peginterferon alfa and ribavirin.

$\mathrm{SAE}$, serious adverse event. 
Flamm S.L. et al.: Telaprevir treatment for HCV in clinical practice

which are more likely to test for IL28B genotype and HCV subtype, only a few charts included test results for IL28B genotype $(30.2 \%)$, and the HCV 1 subtype was unknown in approximately $18 \%$ of patients. The limitations of obtaining these data highlight the variance in the types and quality of information recorded by clinicians, as well as the lack of a standardized approach in collecting such information. Lastly, while general comparisons were made between chart review data from the clinical practice group and the pooled data from the phase 3 studies of telaprevir, no statistical analyses were performed, as direct comparisons cannot be made between studies that were conducted at different times, with different levels of rigor, and not designed in advance with the intent to demonstrate differences.

\section{Conclusions}

This retrospective study of real-life patients with chronic HCV infection treated with telaprevir provides insight into baseline patient demographics and clinical characteristics, as well as dosing and safety and tolerability profiles for telaprevir-based treatment in clinical practice settings. Compared to the phase 3 studies, in clinical practice, the composite baseline patient profiles were considered more difficult to treat and anemia was reported twice as often. AEs led to discontinuation of treatment in $10 \%$ of patients, suggesting that AEs experienced by patients in a clinical setting are generally manageable. Findings from this study complement those reported from rigorous, randomized controlled studies with telaprevirbased treatment and provide a general assessment of the similarities and/or differences between patients from the phase 3 studies of telaprevir and those treated with telaprevir in real-world clinical practice. Treatment with telaprevirbased regimens should be individualized based on both clinical practice observations and data from randomized controlled studies.

\section{Writing assistance}

Medical writing and coordination support were provided by Erika D. Reynoso, PhD, who was an employee of Vertex Pharmaceuticals Incorporated and may own stock or stock options in that company. Medical writing, editorial, and graphic design support was provided by Bina J. Patel, PharmD, of Peloton Advantage and was funded by Vertex Pharmaceuticals Incorporated.

\section{Conflict of interest}

This study was sponsored by Vertex Pharmaceuticals Incorporated. The sponsors were involved in study design, data collection and analysis, decision to publish, and preparation of the manuscript.

S.L. Flamm has served as a remunerated consultant/ lecturer for Bristol-Myers Squibb, AbbVie, Gilead Sciences, Merck \& Co., and Vertex Pharmaceuticals Incorporated, and has received grants/research support from AbbVie, Achillion, Anadys Pharmaceuticals, Bristol-Myers Squibb, Boehringer Ingelheim, Gilead Sciences, Janssen Pharmaceuticals, Merck \& Co., Pfizer, and Vertex Pharmaceuticals Incorporated. He has also served as an advisor or review committee member for Gilead Sciences, Merck \& Co., and Vertex Pharmaceuticals Incorporated. PJ Pockros has served as a remunerated consultant for Boehringer Ingelheim, Bristol-Myers Squibb, Genentech, Gilead Sciences, Janssen Pharmaceuticals, Merck \& Co., Novartis, and Vertex Pharmaceuticals Incorporated, and has received research grants from Abbott, Bristol-Myers Squibb, Boehringer Ingelheim, Genentech, Gilead Sciences, Janssen Pharmaceuticals, Merck \& Co., and Vertex Pharmaceuticals Incorporated. He has also received fees for non-CME services from Genentech, Merck \& Co., and Vertex Pharmaceuticals Incorporated. L. Bengtsson is an employee of Vertex Pharmaceuticals Incorporated and may own stock or stock options in that company. M. Friedman was an employee of Vertex Pharmaceuticals Incorporated at the time of this research and may own or may have owned stock or stock options in that company.

\section{Author contributions}

Study designing (SLF, PJP, LB, MF), collection and assembly of data(LB, MF), data analysis and interpretation (SLF, PJP, LB, MF), manuscript preparation (SLF, PJP, MF), critical review of manuscript (SLF, PJP, LB, MF), final approval of manuscript (SLF, PJP, LB, MF)

\section{Acknowledgments}

The authors thank Bryan Still, Mary Helen Broussard, Andrea Scherschel, NP, and Jill Chang from the Scripps Clinic for their assistance to Dr. Pockros during the study, and Vikas Gulati, of Vertex Pharmaceuticals Incorporated, for clinical data management. Medical writing and coordination support were provided by Erika D. Reynoso, PhD, formerly of Vertex Pharmaceuticals Incorporated. Vikas Gulati is an employee of Vertex Pharmaceuticals, and Erika D. Reynoso was an employee of Vertex Pharmaceuticals Incorporated. Both may own stock or stock options in that company. Medical writing, editorial, and graphic design support was provided by Bina J. Patel, PharmD, of Peloton Advantage and was funded by Vertex Pharmaceuticals Incorporated.

The following investigators also participated in this study: Michael Galambos (Digestive Healthcare of Georgia, Atlanta, GA); Stevan Gonzalez (Baylor All Saints Medical Center, Fort Worth, TX); Mark Jonas (Consultants for Clinical Research, Cincinnati, $\mathrm{OH}$ ); Vishal Patel (Temple University, Philadelphia, PA); and Vinod Rustgi (Metropolitan Research, Fairfax, VA).

\section{Conflict of interest}

This study was sponsored by Vertex Pharmaceuticals Incorporated. The sponsors were involved in study design, data collection and analysis, decision to publish, and preparation of the manuscript.

S.L. Flamm has served as a remunerated consultant/ lecturer for Bristol-Myers Squibb, AbbVie, Gilead Sciences, Merck \& Co., and Vertex Pharmaceuticals Incorporated, and has received grants/research support from AbbVie, Achillion, Anadys Pharmaceuticals, Bristol-Myers Squibb, Boehringer Ingelheim, Gilead Sciences, Janssen Pharmaceuticals, Merck \& Co., Pfizer, and Vertex Pharmaceuticals Incorporated. He has also served as an advisor or review committee member for Gilead Sciences, Merck \& Co., and Vertex Pharmaceuticals Incorporated. PJ Pockros has served as a remunerated consultant for Boehringer Ingelheim, Bristol-Myers Squibb, 
Genentech, Gilead Sciences, Janssen Pharmaceuticals, Merck \& Co., Novartis, and Vertex Pharmaceuticals Incorporated, and has received research grants from Abbott, Bristol-Myers Squibb, Boehringer Ingelheim, Genentech, Gilead Sciences, Janssen Pharmaceuticals, Merck \& Co., and Vertex Pharmaceuticals Incorporated. He has also received fees for non-CME services from Genentech, Merck \& Co., and Vertex Pharmaceuticals Incorporated. L. Bengtsson is an employee of Vertex Pharmaceuticals Incorporated and may own stock or stock options in that company. M. Friedman was an employee of Vertex Pharmaceuticals Incorporated at the time of this research and may own or may have owned stock or stock options in that company.

\section{Author contributions}

Study designing (SLF, PJP, LB, MF), collection and assembly of data(LB, MF), data analysis and interpretation (SLF, PJP, LB, $M F)$, manuscript preparation (SLF, PJP, MF), critical review of manuscript (SLF, PJP, LB, MF), final approval of manuscript $(\mathrm{SLF}, \mathrm{PJP}, \mathrm{LB}, \mathrm{MF})$

\section{References}

[1] Glasser SP, Salas M, Delzell E. Importance and challenges of studying marketed drugs: what is a phase IV study? Common clinical research designs, registries, and self-reporting systems. J Clin Pharmacol 2007;47: 1074-1086, doi: 10.1177/0091270007304776.

[2] Garrison LP, Jr., Neumann PJ, Erickson P, Marshall D, Mullins CD. Using realworld data for coverage and payment decisions: the ISPOR Real-World Data Task Force report. Value Health 2007;10:326-335, doi: 10.1111/j.15244733.2007.00186.x

[3] Olsson J, Terris D, Elg M, Lundberg J, Lindblad S. The one-person randomized controlled trial. Qual Manag Health Care 2005;14:206-216, doi: 10.1097/ 00019514-200510000-00002.

[4] Healey M, Deverka P. Panel 1: methodological issues in pharmacoeconomic evaluations-clinical studies. Value Health 1999;2:73-77, doi: 10.1046/ j.1524-4733.1999.02202.x.

[5] Fusfeld L, Aggarwal ], Dougher C, Vera-Llonch M, Bubb S, Donepudi M, et al. Assessment of motivating factors associated with the initiation and completion of treatment for chronic hepatitis C virus (HCV) infection. BMC Infect Dis 2013;13:234, doi: 10.1186/1471-2334-13-234.

[6] Fried MW, Reddy KR, Di Bisceglie AM, Jensen DM, Jacobson IM, Sulkowsk MS, et al. HCV-TARGET: a longitudinal, observational study of North
American patients with chronic hepatitis $\mathrm{C}(\mathrm{HCV})$ treated with boceprevir or telaprevir. J Hepatol 2013;58 (suppl 1):S335.

[7] Solomon M, Bonafede M, Pan K, Wilson K, Beam C, Chakravarti P, et al. Direct medical care costs among pegylated interferon plus ribavirin-treated and untreated chronic hepatitis C patients. Dig Dis Sci 2011;56:3024-3031, doi: 10.1007/s10620-011-1802-z.

[8] Chew KW, Allen SA, Taylor LE, Rich JD, Feller E. Treatment outcomes with pegylated interferon and ribavirin for male prisoners with chronic hepatitis $C$. J Clin Gastroenterol 2009;43:686-691.

[9] Lo Re V, III, Teal V, Localio AR, Amorosa VK, Kaplan DE, Gross R. Relationship between adherence to hepatitis $C$ virus therapy and virologic outcomes: a cohort study. Ann Intern Med 2011;155:353-360, doi: 10.7326/0003-4819155-6-201109200-00003.

[10] Fontaine H, Hezode C, Dorival C, Larrey D, Zoulim F, de Ledinghen V, et al. SVR12 rates and safety of triple therapy including telaprevir or boceprevir in 221 cirrhotic non responders treated in the French Early Access Program (ANRS CO20-CUPIC). J Hepatol 2013;58 (suppl 1):S27.

[11] Vera-Llonch M, Martin M, Aggarwal ], Donepudi M, Bayliss M, Goss T, et al. Health-related quality of life in genotype 1 treatment-naive chronic hepatitis $C$ patients receiving telaprevir combination treatment in the ADVANCE study. Aliment Pharmacol Ther 2013;38:124-133, doi: 10.1111/apt.12354.

[12] Jacobson IM, McHutchison JG, Dusheiko G, Di Bisceglie AM, Reddy KR, Bzowej $\mathrm{NH}$, et al. Telaprevir for previously untreated chronic hepatitis $\mathrm{C}$ virus infection. N Engl J Med 2011;364:2405-2416.

[13] Sherman KE, Flamm SL, Afdhal NH, Nelson DR, Sulkowski MS, Everson GT, et al. Response-guided telaprevir combination treatment for hepatitis $C$ virus infection. N Engl J Med 2011;365:1014-1024.

[14] Zeuzem S, Andreone P, Pol S, Lawitz E, Diago M, Roberts S, et al. Telaprevir for retreatment of HCV infection. N Engl J Med 2011;364:2417-2428.

[15] Incivek [package insert]. Cambridge, MA: Vertex Pharmaceuticals Incorporated, 2013

[16] Hézode C, Fontaine H, Dorival C, Larrey D, Zoulim F, Canva V, et al. Triple therapy in treatment-experienced patients with HCV-cirrhosis in a multicentre cohort of the French Early Access Programme (ANRS CO20-CUPIC) NCT01514890. J Hepatol 2013;59:434-441, doi: 10.1016/ j.jhep.2013.04.035.

[17] Afdhal N, Reau N, Everson GT, Morelli G, Lok AS, Sherman KE, et al. Safety and efficacy of telaprevir (TVR) or boceprevir (BOC) in patients with cirrhosis: interim results of a longitudinal, observational study. Hepatology 2013;58 (suppl 4):1103a-1104a.

[18] Di Bisceglie AM, Kuo A, Rustgi VK, Sulkowski MS, Sterling RK, Stewart T, et al. Virologic outcomes and adherence to treatment algorithms in a longitudina study of patients with chronic hepatitis $C$ treated with boceprevir (BOC) or telaprevir (TVR) in the United States (HCV-TARGET). Hepatology 2013;58 (suppl 4):227a-228a.

[19] Hess DR. Retrospective studies and chart reviews. Respir Care 2004;49: 1171-1174.

[20] Gearing RE, Mian IA, Barber J, Ickowicz A. A methodology for conducting retrospective chart review research in child and adolescent psychiatry. J Can Acad Child Adolesc Psychiatry 2006;15:126-134. 